\title{
Objetivos de Desarrollo Sostenible y Responsabilidad Social Universitaria integrados en un modelo de Planeación Estratégica
}

\author{
Sustainable Development Objectives and University Social Responsibility integrated in \\ a Strategic Planning model
}

Saraí Aguilar-Barojas

Universidad Juárez Autónoma de Tabasco

mtra_sarai@hotmail.com

Tabasco, México
Cómo referenciar:

Aguilar Barojas, S. (2022). Objetivos de Desarrollo Sostenible y Responsabilidad Social Universitaria integrados en un modelo de Planeación Estratégica

Emerging Trends in Education, (4)8A, 125-141

https://doi.org/10.19136/etie.a4n8A.4780

Disponible en:

https://revistas.ujat.mx/index.php/emerging

DOI:

https://doi.org/10.19136/etie.a4n8A.4780

Recibido: Aceptado:

Publicado:

07/10/2021

$17 / 12 / 2021$

05/01/2022

\section{Resumen:}

Los Objetivos de Desarrollo Sostenible (ODS) son un llamado de la Organización de las Naciones Unidas (ONU) para que todos los países adopten las medidas necesarias para mejorar diversas situaciones que han deteriorado el ambiente y atrasado el desarrollo de los pueblos, la paz y la prosperidad. El paso de los jóvenes por la universidad es un momento crucial de sensibilizacion y educación en estos temas, para lo cual se propone un modelo de Planeación estratégica que integra a los ODS con la Responsabilidad Social Universitaria (RSU), con el fin de garantizar su abordaje y cumplimiento. El modelo representado es dinámico y tiene tres planos: en el centro están colocados los ODS, la misión, visión y valores institucionales enlazados con la RSU; en un segundo plano están los elementos de la planeación (objetivos, estrategias y las metas); el tercer plano considera la evaluación de la estructura, los procesos y los resultados. Los tres planos convergen en las cuatro funciones sustantivas universitarias. Un segundo esquema muestra las interacciones entre los elementos considerados. La adaptación de este modelo permite señalar y mantener centrados a los ODS para sumar las universidades al desafío de la meta mundial planteada al 2030.

Palabras clave: Modelo; responsabilidad social universitaria; objetivos de desarollo sostenible; planeación estratégica; educación superior.

\begin{abstract}
:
The Sustainable Development Goals (SDGs) are a call from the United Nations Organization for all countries to adopt the necessary measures to improve various situations that have deteriorated the environment and delayed the development of peoples, peace and prosperity. In this context, the passage of young people through the university is a crucial moment of awareness and education on these issues for which a strategic planning model is proposed that integrates the SDGs with University Social Responsibility (RSU) in order to guarantee its approach and compliance. The model presented in a first diagram is dynamic and has three planes. In the center are the SDGs in addition to the mission, vision and institutional values linked to USR. In a second plane are the elements of planning, such as objectives, strategies and goals. A third plane considers the evaluation of the structure, the processes and the results. The three planes converge in the four university substantive functions. A second diagram shows the interactions between the elements considered. The adaptation of this model makes it possible to point out and keep focused on the SDGs to add universities to the challenge of the world goal set for 2030 .
\end{abstract}

Keywords: Model; university social responsibility; sustainable development goals; strategic planning; higher education. 


\section{Objetivos de Desarrollo Sostenible y Responsabilidad Social Universitaria integrados en un modelo de Planeación Estratégica}

\section{| Introducción}

La sostenibilidad es un tema que ha sido puesto por la Organización de las Naciones Unidas (ONU) en la agenda de todos los países a través de los 17 Objetivos de Desarrollo Sostenible (ODS), los cuales necesitan de un espíritu de colaboración para implementar las mejores estrategias que mejoren la vida de las generaciones futuras de manera sostenible. Conocidos como Objetivos Mundiales, al considerarse como un llamado para que todos los países adopten medidas que mitiguen o pongan fin a la pobreza, protejan al planeta de los efectos adversos causados por la sobre explotación y la generación de gases que continúan modificando el clima, además que se garantice a las poblaciones la paz y prosperidad a la que tienen derecho (Organización de las Naciones Unidas [ONU], 2016a).

Ante este panorama, la universidad se convierte en un valioso instrumento para el mejoramiento de las sociedades, al encontrarse en el núcleo de los cambios y los riesgos que estas enfrentan, donde su vocación social cobra relevancia dado que todas, en mayor o menor medida, han sido penetradas por las características de la globalización, que guía a las universidades a formar estudiantes de clase mundial compitiendo entre sí con base en estándares internacionales convertidos en un fin en sí mismos, generando tensión entre el modelo tradicional de cooperación y la competencia por lograr el reconocimiento en rankings (Ramírez, 2017).

En este contexto, la esperanza es actuar proactivamente e implementar estrategias policéntricas que, además de difundirse entre los ciudadanos, generen confianza y promuevan cambios éticos en lo personal, ya que todos son parte del problema y también parte de la solución. De ahí que los comportamientos deben de cambiar, con base en los pilares fundamentales de las relaciones sociales, que son libertad y responsabilidad (Castilla, 2015).

Tomando en cuenta lo anterior, el paso de los estudiantes por la universidad se considera un periodo valioso para que se formen con valores éticos de manera cotidiana, desarrollen la capacidad y sensibilidad para identificar principios éticos universales, y más tarde los apliquen en su actuar profesional y personal. Esto puede lograrse si durante su formación universitaria se integra la teoría con la práctica, con el fin de lograr una adecuada identificación, comprensión y enfrentamiento de situaciones con implicaciones éticas (Winkler et al., 2012).

Desde hace más de una década, estos temas se han reflexionado en foros de gran importancia como la Conferencia Mundial sobre Educación Superior celebrada en la sede de la Organización de las Naciones Unidas para la Educación, la Ciencia y la Cultura (UNESCO) en 2009, donde se destacó que la educación superior tiene una gran responsabilidad en los contenidos curriculares, éticos y en los valores que se transmiten y que requiere de pertinencia académica y social para dar tratamiento a 
los desafíos mundiales como la seguridad alimentaria, la gestión de los recursos naturales, el diálogo intercultural, para lo cual debe contraer un verdadero compromiso con la sociedad a través de la Responsabilidad Social Universitaria (RSU) (Organización de las Naciones Unidas para la Educación, la Ciencia y la Cultura [UNESCO], 2009).

Específicamente en Latinoamérica, los esfuerzos teóricos y prácticos para construir un nuevo paradigma que fundamente la gestión de los impactos negativos que tienen las Instituciones de Educación Superior (IES) durante la ejecución de sus funciones sustantivas, tuvo su origen a inicios de los años 2000 y ha cobrado fuerza gracias a la creación de organizaciones como El Observatorio Regional de Responsabilidad Social para América Latina y el Caribe (ORSALC), cuyos consultores atienden el marco de actuación latinoamericano de la RSU desde su creación por la UNESCO en 2012.

En México, la RSU en un inicio se consideraba la mejor estrategia para conducir la transformación necesaria en las instituciones de educación superior; esto quedó establecido en el primer encuentro de RSU convocado por ORSALC en 2014, donde las universidades reflexionaron sobre cómo hacer para adoptar políticas sobre la RSU en su ambiente cotidiano. Dos años después, en 2016, se creó el Observatorio Mexicano de Responsabilidad Social Universitaria (OMERSU), organismo apoyado por la Asociación Nacional de Universidades e Instituciones de Educación Superior (ANUIES) y varias universidades cofundadoras, teniendo entre otros, el objetivo de promover la RSU entre las IES.

En las reflexiones realizados por diferentes actores universitarios en encuentros convocados por las organizaciones mencionadas se ha identificado a la RSU como una política, visión, filosofía, estrategia o acción. En todas estas concepciones, finalmente se trata de que promueva el paso del discurso a la acción para formar profesionales integrales comprometidos con la sociedad, de ahí que, es muy importante que la RSU encuentre un lugar dentro de la planeación y gestión estratégica para que se ejecute y evalúe con indicadores pertinentes y se pueda conocer el impacto que tiene tanto en la formación de los estudiantes como en la sociedad (Navarro, 2011).

Además de lo anterior, es necesario que el modelo adoptado en una IES tome a los ODS como guía de su plan rector y logre una formación de los estudiantes, atendiendo la Responsabilidad Social con que debe actuar cotidianamente. De esta manera, los ODS y la RSU quedarían unidos a través del modelo de planeación que utilice la institución, asegurando el cumplimiento de ambos.

Una revisión de los modelos de RSU que han surgido en la última década en Latinoamérica muestra el enriquecimiento que ha tenido el tema para dar paso a modelos cada vez más robustos, que permitan fundamentar el rumbo a seguir, proponer los elementos a considerar, posibilitar su evaluación y establecer las relaciones en esquemas que puedan ser fácilmente interpretados.

En la tabla 1 se presenta un resumen del análisis de 14 modelos propuestos en varios países latinoamericanos por organizaciones e investigadores universitarios. En todos ellos se mencionan las funciones sustantivas de las IES y se observa que en 2013 una universidad en México ya consideraba a la RSU como un marco de políticas operativas; esta concepción la han apropiado y mantenido varias universidades hasta la actualidad. Sin embargo, a pesar de que varios modelos mencionan la sustentabilidad y el medio ambiente, es a partir de 2019 que algunos modelos de RSU la vinculan abiertamente con los ODS y presentan esquemas que indican su relación y direccionamiento 
Tabla 1

Características de los modelos de RSU propuestos en la útima década en Latinoamérica

\begin{tabular}{|c|c|c|c|c|}
\hline Año & $\begin{array}{c}\text { País/Región - Ins- } \\
\text { titución }\end{array}$ & Autor & Características & Observaciones \\
\hline 2011 & \begin{tabular}{l} 
Ecuador - Univer- \\
sidad Politécnica \\
Salesianar Sede \\
\multicolumn{2}{c}{ Cuenca }
\end{tabular} & $\begin{array}{l}\text { Calle-Ramírez \& } \\
\text { Santacruz-Mon- } \\
\text { cayo (2011) }\end{array}$ & $\begin{array}{l}\text { El modelo propuesto está centrado en un es- } \\
\text { quema de cuatro pasos: 1) compromiso, 2) au- } \\
\text { todiagnóstico participativo al interior y exterior } \\
\text { (aspectos organizacional, educativo, cognitivo y } \\
\text { social), 3) cumplimiento y 4) rendición de cuen- } \\
\text { tas }\end{array}$ & No hay esquema del modelo \\
\hline 2013 & $\begin{array}{l}\text { México - Univer- } \\
\text { sidad Autónoma } \\
\text { Metropolitana } \\
\text { (UAM) (Unidad } \\
\quad \text { Cuajimalpa) }\end{array}$ & UAM (2013) & $\begin{array}{l}\text { El modelo se considera como un marco de po- } \\
\text { líticas operativas (ámbito social, económico y } \\
\text { ambiental) alineado con el PDI en las dimen- } \\
\text { siones de formación, investigación, gestión y } \\
\text { vinculación. }\end{array}$ & No hay esquema del modelo \\
\hline 2013 & $\begin{array}{l}\text { México - Univer- } \\
\text { sidad Autónoma } \\
\text { de Nuevo León } \\
\text { (UANL) }\end{array}$ & Cruz et al (2013) & $\begin{array}{l}\text { Adapta elementos de RS presentados en la nor- } \\
\text { ma ISO } 26000 \text { a los que añade elementos de } \\
\text { transparencia. Las dimensiones que considera } \\
\text { son docencia, investigación, gestión y exten- } \\
\text { sión unidos por principios y valores }\end{array}$ & $\begin{array}{c}\text { Diagrama de involucrados con base en la } \\
\text { norma ISO } 26000 \text { y esquema de las cuatro } \\
\text { dimensiones }\end{array}$ \\
\hline 2014 & $\begin{array}{c}\text { Latinoamérica }- \\
\text { UNIVERSIA }\end{array}$ & Vallaeys (2014) & $\begin{array}{l}\text { La RSU se considera como una política de ges- } \\
\text { tión universitaria y una estrategia de mejora } \\
\text { continua que permite legitimar al conocimien- } \\
\text { to y a la universidad }\end{array}$ & $\begin{array}{c}\text { Esquema que presenta cuatro impactos en } \\
\text { los ejes organizacional y académico }\end{array}$ \\
\hline 2014 & $\begin{array}{l}\text { Ecuador - Univer- } \\
\text { sidad Técnica Par- } \\
\text { ticular de Loja }\end{array}$ & $\begin{array}{l}\text { Valarezo \& Túñez } \\
\text { (2014) }\end{array}$ & $\begin{array}{l}\text { Construye de manera grupal una definición de } \\
\text { RSU con el objetivo de lograr un desarrollo hu- } \\
\text { mano sostenible. Integra cuatro dimensiones } \\
\text { (académica, investigación, extensión y gestión) } \\
\text { en los ejes de ética, transparencia, diálogos, } \\
\text { vínculos y rendición de cuentas }\end{array}$ & $\begin{array}{c}\text { Presenta un esquema para la comunicación } \\
\text { en la gestión de la RSU }\end{array}$ \\
\hline 2014 & $\begin{array}{l}\text { México - Universi- } \\
\text { dad Autónoma de } \\
\text { Yucatán (UADY) }\end{array}$ & UADY (2014) & $\begin{array}{l}\text { El modelo considera cuatro dimensiones (for- } \\
\text { mación profesional y ciudadana, gestión social } \\
\text { del conocimiento, gestión responsable y parti- } \\
\text { cipación social) y las estrategias para cada una } \\
\text { de las dimensiones (social, económico y am- } \\
\text { biental) donde impacta }\end{array}$ & $\begin{array}{c}\text { Esquema del modelo integrado por cuatro } \\
\text { dimensiones y los ámbitos social, económi- } \\
\text { co y ambiental donde impactan las funcio- } \\
\text { nes universitarias }\end{array}$ \\
\hline 2016 & $\begin{array}{l}\text { México - Universi- } \\
\text { dad Autónoma de } \\
\text { Chihuahua (UACH) }\end{array}$ & $\begin{array}{l}\text { De Dios-Pérez \& } \\
\text { Vallaeys (2016) }\end{array}$ & $\begin{array}{c}\text { El modelo parte de la Visión y Misión institu- } \\
\text { cional que orientan a cuatro ejes rectores del } \\
\text { Plan de Desarrollo Universitario (Gestión admi- } \\
\text { nistrativa y ambientalmente responsable, Pro- } \\
\text { ducción del conocimiento (investigación), Par- } \\
\text { ticipación social (Extensionismo) y Formación } \\
\text { socialmente responsable de los estudiantes } \\
\text { (Plan académico) }\end{array}$ & $\begin{array}{c}\text { Esquema del modelo utilizado para la elabo- } \\
\text { ración del plan de Desarrollo Institucional. } \\
\text { Identifican una secuencia descendente que } \\
\text { parte de la Visión y Misión }\end{array}$ \\
\hline 2017 & $\begin{array}{l}\text { México - Asocia- } \\
\text { ción Nacional de } \\
\text { Facultades y Es- } \\
\text { cuelas de Conta- } \\
\text { duría y Adminis- } \\
\text { tración (ANFECA) }\end{array}$ & ANFECA (2017) & $\begin{array}{l}\text { El modelo de RSU tiene cuatro principios éti- } \\
\text { cos, además de la participación ciudadana y } \\
\text { la sustentabilidad. Considera que para hacerla } \\
\text { tangible es mediante la incorporación de bue- } \\
\text { nas prácticas. Lo aplican para evaluar la RSU de } \\
\text { las Facultades y Escuelas de Contaduría y Ad- } \\
\text { ministración }\end{array}$ & $\begin{array}{l}\text { Presenta un esquema cuyo centro es la Mi- } \\
\text { sión, la Visión y los valores. Las buenas prác- } \\
\text { ticas evaluadas con indicadores de gestión }\end{array}$ \\
\hline 2017 & $\begin{array}{l}\text { Colombia - Univer- } \\
\text { sidad de Tolima }\end{array}$ & $\begin{array}{l}\text { Uribe-Macías } \\
\text { (2017) }\end{array}$ & $\begin{array}{l}\text { Considera a la RSU una mística y una política. } \\
\text { El modelo está orientado a satisfacer los inte- } \\
\text { reses de los diferentes stakeholders, basado } \\
\text { desde el origen de los procesos y finaliza con su } \\
\text { satisfacción. Propone la creación de una políti- } \\
\text { ca específica de gestión en la universidad }\end{array}$ & $\begin{array}{l}\text { El modelo esquematizado interrelaciona } \\
\text { los procesos y su interacción dentro de las } \\
\text { tres funciones sustantivas de la universidad: } \\
\text { docencia, investigación y proyección social. } \\
\text { Presenta un segundo esquema de planea- } \\
\text { ción del modelo }\end{array}$ \\
\hline 2019 & $\begin{array}{l}\text { Colombia-Asocia- } \\
\text { ción de Universi- } \\
\text { dades Confinadas } \\
\text { a la Compañía de } \\
\text { Jesús en América } \\
\text { Latina (AUSJAL) }\end{array}$ & $\begin{array}{l}\text { Forero-Jiménez } \\
\qquad(2019)\end{array}$ & $\begin{array}{l}\text { Plantea que el modelo de RSU debe ser visto } \\
\text { como un proyecto institucional, por lo que es- } \\
\text { tablece los pasos para su diseño, la creación de } \\
\text { políticas y su divulgación }\end{array}$ & $\begin{array}{l}\text { El modelo esquematiza las características } \\
\text { de transversalidad e interdisciplinariedad } \\
\text { en los planes y proyectos de docencia, pro- } \\
\text { yección social, investigación, gestión, medio } \\
\text { ambiente y posconflicto }\end{array}$ \\
\hline 2019 & $\begin{array}{l}\text { Perú - Universidad } \\
\text { San Ignacio de Lo- } \\
\text { yola (USIL) }\end{array}$ & USIL (2018) & $\begin{array}{l}\text { El modelo de RSU tiene tres niveles, estratégico, } \\
\text { institucional y operativo y cada uno correspon- } \\
\text { de a un ámbito dentro de la organización. Su- } \\
\text { pone una aplicación transversal }\end{array}$ & $\begin{array}{c}\text { El esquema presenta los tres niveles, estra- } \\
\text { tégico, institucional y operativo (gestión } \\
\text { académica y de investigación, gestión am- } \\
\text { biental corporativa y gestión del impacto } \\
\text { social). Se espera que el nivel institucional } \\
\text { impacte en los } 8 \text { ODS priorizados: } 2,4,8,9 \text {, } \\
10,12,13 \text { y } 17\end{array}$ \\
\hline
\end{tabular}




\begin{tabular}{|c|c|c|c|c|}
\hline Año & $\begin{array}{l}\text { País/Región - Ins- } \\
\text { titución }\end{array}$ & Autor & Características & Observaciones \\
\hline 2019 & $\begin{array}{l}\text { Chile - Universi- } \\
\text { dad de Chile }\end{array}$ & $\begin{array}{l}\text { Severino- Gonzá- } \\
\text { lez (2019) }\end{array}$ & $\begin{array}{l}\text { El modelo tiene el propósito de promover la } \\
\text { RSU y explica cómo las actividades de cada uno } \\
\text { de sus cuatro componentes trabajan para pro- } \\
\text { mover la RS y la sustentabilidad articulando la } \\
\text { vinculación y sociedad, formación curricular, } \\
\text { gestión organizacional y difusión del conoci- } \\
\text { miento }\end{array}$ & $\begin{array}{l}\text { De manera gráfica el modelo interrelacio- } \\
\text { na la docencia, investigación, extensión y } \\
\text { gestión. Presenta a la sustentabilidad como } \\
\text { valor fundamental que rodea los cuatro ám- } \\
\text { bitos }\end{array}$ \\
\hline 2019 & $\begin{array}{l}\text { Latinoamérica - } \\
\text { Unión de Respon- } \\
\text { sabilidad Social } \\
\text { Universitaria } \\
\text { noati- } \\
\text { noamericana (UR- } \\
\text { SULA) }\end{array}$ & URSULA (2019) & $\begin{array}{l}\text { El modelo vincula a la universidad con el terri- } \\
\text { torio y la sociedad civil. Mantiene como refe- } \\
\text { rencia a los ODS y la agenda } 2030\end{array}$ & $\begin{array}{l}\text { Introduce un esquema donde compara los } \\
\text { modelos } 2009 \text { y } 2019 \text { donde presenta los } \\
\text { ODS y las } 121 \text { metas de diseño socialmente } \\
\text { responsable }\end{array}$ \\
\hline 2021 & $\begin{array}{c}\text { Latinoamérica } \\
\text { URSULA }\end{array}$ & Vallaeys (2021) & $\begin{array}{l}\text { Define a la RSU en términos de gestión de im- } \\
\text { pactos y la articula con una filosofía de calidad }\end{array}$ & $\begin{array}{c}\text { Presenta un esquema del itinerario de la RSU } \\
\text { con un enfoque Heurístico transformador } \\
\text { que apunta hacia los ODS }\end{array}$ \\
\hline
\end{tabular}

Nota: Elaboración propia en base a los autores citados.

\section{| Aspectos Teóricos}

\section{Los Objetivos de Desarrollo Sostenible}

La Organización de Naciones Unidas (ONU), tiene como principal objetivo el desarrollo social de los pueblos, donde la pobreza, la industrialización, la salud, el cambio climático, la sostenibilidad de los ecosistemas, la democracia, las desigualdades, la paz y la justicia, son temas que trascienden a la generación actual y que, si no se atienden, ponen en riesgo el futuro de los pueblos.

Como estrategia mundial para contrarrestar las desventajas de los países menos desarrollados, en enero de 2016 la ONU (2016a) lanzó 17 Objetivos para el Desarrollo Sostenible (ODS) 2015-2030 concretados en 169 metas (figura 1)

\section{Figura 1}

Objetivos de Desarrollo Sostenible



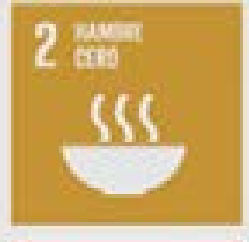
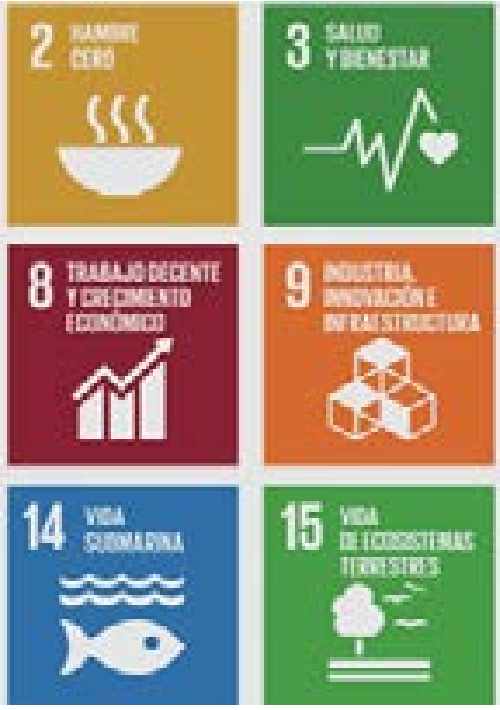
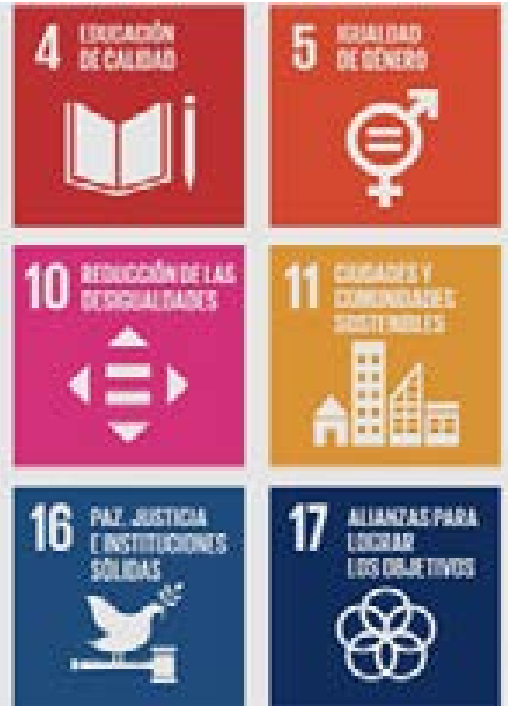

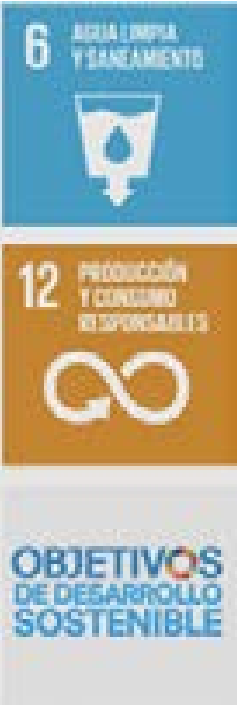

Nota: ONU (2016a). 
En ellos se identifican los efectos desencadenantes del La Responsabilidad Social Universitaria

cambio climático, de ahí que los países desarrollados tienen mucho que hacer para lograr un desarrollo sostenible, que la ONU define como "el desarrollo capaz de satisfacer las necesidades del presente sin comprometer la capacidad de las futuras generaciones para satisfacer sus propias necesidades". Para lograr esto es necesario atender tres elementos fundamentales estrechamente relacionados entre sí: el crecimiento económico de todos los pueblos, la inclusión social en su más amplia acepción y la protección del medio ambiente en todo el planeta (Organización de las Naciones Unidas [ONU], 2016b).

\section{El impacto de los enfoques de la educación universitaria}

A los retos socio económicos y ambientales identificados a nivel mundial se añaden los derivados por el proceso de formación universitaria, el cual impacta no solo a los estudiantes que viven el proceso, sino que se producen otros más que han sido agrupados en cuatro: los impactos de la propia institución, tanto al interior con su gestión organizacional, como hacia el exterior sobre todo en el tema ambiental; los impactos derivados de la formación impartida a los estudiantes; los impactos que se producen con el tipo de investigación producida y los impactos que se generan con su participación social en su anclaje territorial (Vallaeys, 2008a).

El análisis de estos impactos permite identificar la necesidad de replantear la formación universitaria que tiene un enfoque netamente mercantilista y, de no ser el caso, reducida a la transmisión de conocimientos a los estudiantes, centrada en áreas de especialización y con desarraigo e insensibilidad a los problemas sociales y ambientales de su propio territorio (Vallaeys, 2014).

Como respuesta a los retos que implican los impactos identificados, emerge la RSU, que cuestiona el quehacer rutinario y limitado de los académicos y administrativos sobre el trabajo que realizan en las IES, el cual debe de estar en sintonía con la sociedad, que sea ejemplo de buen gobierno con sus empleados y estudiantes, ofertando programas actualizados y aplicados con metodologías que desarrollen el espíritu crítico de los jóvenes para que cuando egresen atiendan los problemas socioeconómicos y ambientales que cada día ponen en riesgo a la población, independientemente de cuál sea la profesión en que se formen.

De acuerdo con las diferentes definiciones que se han propuesto, la RSU puede ser vista de diferentes maneras, como la capacidad institucional para poner en práctica un conjunto de principios (Universidad Construye País, 2002), una política de mejora continua (Vallaeys, 2008b), la habilidad y efectividad que tienen las instituciones para responder a las necesidades de la sociedad (Asociación de Universidades Confiadas a la Compañía de Jesús en América Latina [AUSJAL], 2009), las acciones alineadas con la visión institucional universitaria (KatayamaOmura, 2014), una visión integradora la identifica como el comportamiento de la comunidad universitaria (Gaete, 2015), o bien, una dimensión estratégica de la universidad (Kouatli, 2019). En estas concepciones se menciona a la sociedad o se hace alusión a ella al referirse al impacto social que produce el quehacer universitario mientras que otras definiciones mencionan explícitamente los términos sustentable, ambiental o sostenible.

Entre las líneas teóricas mencionadas destaca la propuesta de Vallaeys (2008a), quien ha profundizado en su estudio e integrado los elementos teóricos necesarios 
para dar solidez a su fundamentación. Siguiendo su línea en este trabajo, la RSU es considerada "una política de calidad ética orientada hacia el desarrollo humano sostenible [...] manteniendo una coherencia entre la misión institucional declarada y la práctica efectiva de la institución" (p.151). Una década después, la considera como "el sistema inmunológico de toda la IES, es decir un proceso integral y transversal que vigile que todo lo que se haga en la institución sea socialmente responsable, evite tener impactos negativos y promueva impactos positivos" (Vallaeys, 2021, p. 33). Y más adelante concreta "al promover los ODS en el territorio de desempeño e incidencia de las IES" (p. 46).

\section{La Planeación Estratégica en las Universidades}

Si se considera a la RSU como la política institucional que debe adoptar la universidad en su actuación, ésta debe permear desde los principios y valores institucionales, continuar su presencia durante la planeación estratégica con el establecimiento de los objetivos, estrategias y metas de las funciones sustantivas, hasta concluir con la evaluación y, de acuerdo con los resultados, realimentar al sistema. Por ello, es muy importante que la RSU encuentre un lugar visible dentro de la planeación y gestión estratégica para que se ejecuten las acciones, evalúen sus resultados y se pueda conocer el impacto que tiene la universidad tanto en la formación de los estudiantes como en la sociedad (Navarro, 2011).

En congruencia, se necesitará diseñar una misión que declare la razón de ser de la universidad y una visión que permita identificar a dónde quiere llegar en el futuro. Ambas consideradas como las piedras angulares del proceso de planeación estratégica de la organización (Hax
\& Majluf, 1984).

Para que una IES se considere socialmente responsable, es necesario que tanto la misión como la visión contengan elementos de RSU explícitos que garanticen su inclusión en los objetivos, estrategias y metas que se diseñen en cada una de las funciones sustantivas de la universidad.

Un análisis realizado a la declaración de principios de IES latinoamericanas reportó la baja inclusión de elementos de RSU en su misión y visión. Aún cuando la Formación Profesional y Ciudadana y la Gestión Social del conocimiento sí se mencionan, casi no se identifican elementos del campus responsable ni la participación social (Gaete \& Álvarez, 2019). Atender estas omisiones resulta fundamental para que las instituciones puedan alinear su filosofía con la operación y evaluación objetiva que realimente los procesos de la organización.

Una filosofía institucional construida de manera cuidadosa y consensada dará dirección a la planeación estratégica, la cual ha sido definida de diversas maneras, desde arte hasta proceso. De su revisión se entiende como una práctica que permita relacionar los resultados que se desean con los medios de lograrlos, esto es, las metas con las estrategias.

De acuerdo con el modelo de la Sociedad Alemana de Cooperación Internacional presentado en la figura 2, el proceso del ciclo estratégico se divide en cinco pasos lineales que inician con el análisis de la situación actual mediante la identificación de las fortalezas, debilidades, oportunidades y amenazas, cuyo análisis constituye el diagnóstico para continuar con la identificación de opciones, elegir las mejores y diseñar las estrategias para integrarlas en el proceso de gestión (Deutsche Gesellschaft für International Zusammenarbeit [GIZ], 2015, p.97). 
Figura 2

Desarrollo de la Estrategia como proceso lineal

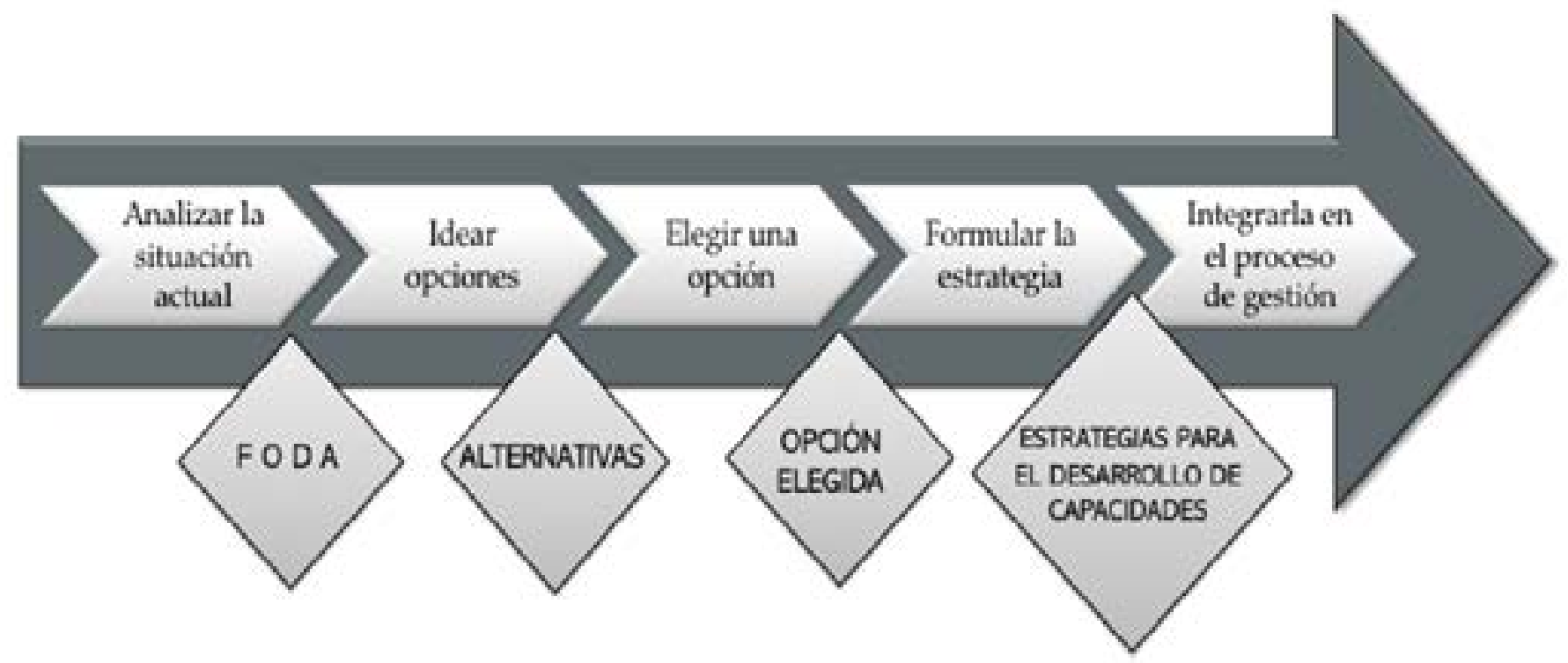

Nota: Deutsche Gesellschaft für International Zusammenarbeit [GIZ], 2015, p.97.

La etapa de evaluación consiste en la recolección, organización y análisis de la información, con resultados que permitirán establecer un juicio de cómo se está llevando a cabo la educación de los jóvenes y los impactos que se tienen al interior y exterior de la institución. Solo después de contrastar lo que se estableció como objetivos y metas, y comparar los avances, se podrá tener un panorama que permita la toma de decisiones.

Vista así, la evaluación es una fase fundamental, no solo en la educación, sino en todas las áreas productivas. Es así que, para evaluar la calidad de los servicios en el campo de la salud desde principio de los años ochenta se introdujo el enfoque teórico propuesto por Avedis Donabedian (1978/2001a), el cual es integral ya que considera la estructura, los procesos y los resultados, transformando los juicios de valor en indicadores para que puedan ser reproducidos, sistematizados e interpretados de la manera más homogénea, en un área tan trascendente como es la atención de los pacientes hospitalizados.
Los indicadores de estructura son los más comúnmente utilizados para evaluar la capacidad o instalaciones disponibles para proporcionar un servicio (preparación de las condiciones para el servicio). Los indicadores de procesos evalúan qué tan bien se está proporcionando el servicio y dan cuenta de la mejora de la calidad (condiciones durante el servicio); mientras que los indicadores de resultados son el reflejo del efecto del servicio. También reflejan lo adecuado de la estructura, así como la validez del proceso (condiciones después del servicio).

La ventaja principal de utilizar indicadores para la evaluación es resumir grandes cantidades de información disponible a elementos unidimensionales fáciles de comparar y entender, mejorando la toma de decisiones. Sin embargo, la integración de las múltiples dimensiones de un sistema o programa dentro de un indicador puede llevar a la incertidumbre y la redundancia de los datos. Debido a lo anterior, es recomendable garantizar que estos últimos sean accesibles y que la frecuencia de 
la medición sea razonable (Comisión Económica para América Latina y el Caribe [CEPAL], 2009)

\section{| Aportaciones}

\section{El modelo de integración ODS-RSU-Planeación Estratégica}

Un modelo implica que se especifiquen tres factores: el fenómeno que constituye el objeto de interés, establecer los atributos de este fenómeno, y los criterios explícitos y normas que coloquen a cada atributo en una escala que refleje el estado en que se encuentra. Por lo anterior y, atendiendo al primer punto, la definición de la RSU debe diseñarse en términos precisos y funcionales, de tal manera que se facilite la identificación de sus atributos, los criterios y normas (Donabedian, 1993/2001b).

Así, para efectos de este trabajo se define a la RSU como el comportamiento de una IES que lleva a cabo una gestión organizacional con enfoque ecológico, comprometida con el bienestar de la comunidad universitaria; la formación integral con pertinencia social de ciudadanos críticos, responsables y solidarios; la producción y difusión de conocimientos útiles para el desarrollo inclusivo y sostenible local, nacional e internacional; la participación activa en el diseño de los programas gubernamentales y de proyectos co-creados con la sociedad; aplicando siempre principios éticos en todas sus funciones, las que articula para impulsar la agenda de los ODS.

Una vez establecido el constructo de RSU y derivado del análisis documental, se seleccionaron los atributos y elementos que se proponen para ser considerados en el modelo de integración presentado en la figura 3.
Figura 3

Modelo de integración ODS-RSU-Planeación Estratégica

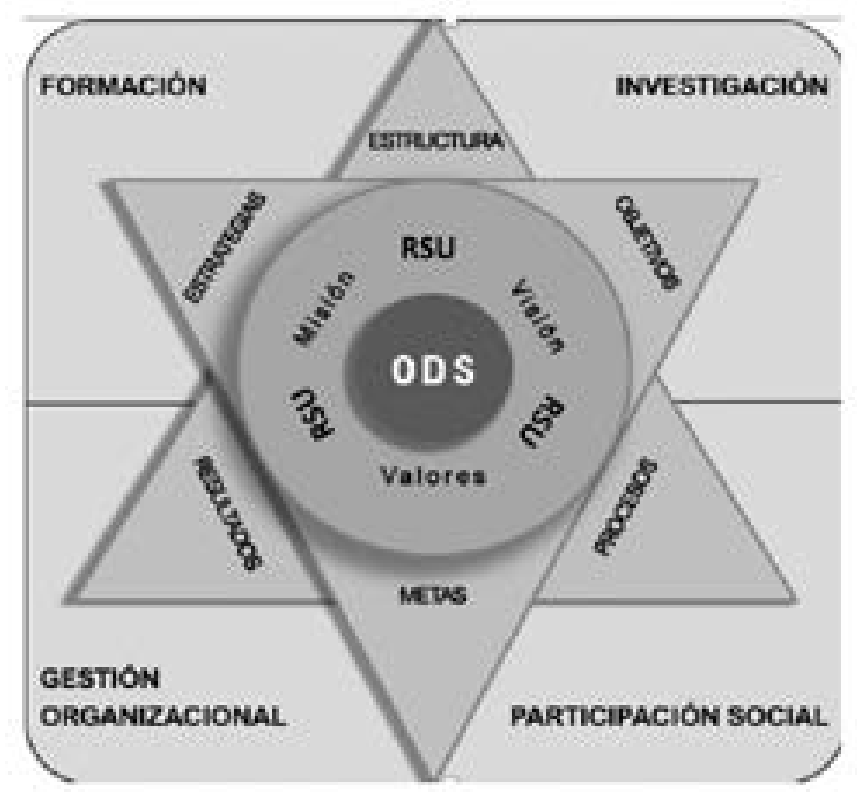

Nota: Elaboración propia.

Explicación de los elementos del modelo

\section{Primer plano}

El centro del modelo. En el centro del modelo se colocaron los 17 ODS como guía del quehacer universitario, pues su objetivo es poner fin a la pobreza en los países menos desarrollados, mitigar la desigualdad y la injusticia, así como enfrentar los estragos del cambio climático a nivel mundial. Esto es congruente con el compromiso contraído por el estado mexicano en septiembre de 2015, cuando se reunieron 193 países miembros de la ONU para aprobar la agenda presentada en el documento llamado Transformar nuestro Mundo: la Agenda 2030 para el Desarrollo Sostenible. Al respecto, el organismo realizó un llamado a sus miembros para desplegar las estrategias que fueran necesarias y lograr hacer frente básicamente a tres temas interconectados entre sí: el desarrollo económico, la inclusión social y la sostenibilidad ambiental (ONU, 2016b). 
Para lograr la agenda al año 2030, se ha reconocido que la educación es el motor más importante para el desarrollo, no solo del objetivo 4 referido a la educación, donde se plantea el "Garantizar una educación inclusiva, equitativa y de calidad [...]", sino para la consecución de los demás ODS (Organización de las Naciones Unidas para la Educación, la Ciencia y la Cultura [UNESCO] \& Instituto Internacional para la Educación Superior en América Latina y el Caribe [IESALC], 2019, p. 3).

Con base en ello, la Conferencia Regional de Educación Superior (CRES) emitió en el principio declarativo No. 7 sobre la educación Superior en América Latina y el Caribe, que ésta "Responde a los Objetivos de Desarrollo Sostenible de forma integrada dada la complejidad social, económica, política, educativa, cultural lingüística, biológica y geográfica de la región de América Latina y el Caribe" (UNESCO \& IESALC, 2019, p. 9).

El logro de los 17 ODS puede ser impulsado por la universidad a través de las cuatro funciones sustantivas que desarrollan, en algunos casos hacia el exterior de la institución, actuando directamente en las comunidades e impactando en los sectores económicos, en otros hacia el interior incidiendo en la comunidad universitaria y en varios de ellos realizando actividades tanto al exterior como al interior de esta.

\section{La Misión, Visión y Valores institucionales}

Alineados con los ODS en el centro del modelo se encuentran la misión, visión y valores de la Universidad que constituyen su declaración de principios al expresar su razón de ser e imagen futura, cuya expresión debe contener los elementos fundamentales del constructo de RSU, además de estar enmarcadas por valores éticos. De esta manera, el ejercicio de planeación estratégica que se derive estará alineado hacia el cumplimiento de esos principios institucionales teniendo en cuenta a la
RSU. En el esquema del modelo propuesto se presentan entretejidos los cuatro elementos mencionados.

\section{Segundo plano}

\section{Objetivos, estrategias y metas}

Una vez establecidas la misión y visión universitarias, para cada una de las dimensiones del quehacer universitario se diseñan los objetivos, las estrategias y metas, que son la triada que indica hacia dónde se dirige la institución, cómo lo hará y qué tanto va a avanzar en la formación, investigación, participación social y la gestión organizacional. Durante este proceso de planeación estratégica, un aspecto clave es la formulación de las estrategias, considerado como un momento orientador que apoya la definición de los objetivos organizacionales, los recursos a utilizar, así como las políticas que orientarán la ejecución del plan.

Para diseñar estrategias que sean exitosas, el ejercicio debe ser realizado por un equipo de personas involucradas con el quehacer académico, administrativo y de investigación de la institución, coordinados por los líderes identificados en cada área.

En las IES, el diseño de las estrategias necesita de la participación de todos los actores para que cada uno, desde su realidad, exprese sus intereses y proponga alternativas de abordaje y solución. Para la recolección de la información, es necesario que se utilicen diferentes técnicas tanto cualitativas para profundizar los casos, como cuantitativas para que los hallazgos se puedan extrapolar a la población estudiada.

Para efectos de explicar este modelo integrador, en la tabla 2 se hacen sugerencias de objetivos y estrategias diseñadas tomando en cuenta elementos de RSU ya expresados en la filosofía institucional y, en congruencia con el modelo, para cada una de las funciones sustantivas 
se incluyen varias estrategias que mencionan explícitamente a los ODS, mientras que otras se desplegarán en acciones que también apoyarán su avance.

Tabla 2

Objetivos y estrategias propuestos para un Modelo de integración ODS-RSU-Planeación Estratégica

\begin{tabular}{|c|c|c|}
\hline Función & Objetivos & Estrategias \\
\hline Formación & $\begin{array}{l}\text { Favorecer el espíritu crítico desarrollo } \\
\text { humano y la responsabilidad ambien- } \\
\text { tal, de estudiantes, egresados y de la } \\
\text { sociedad en general. }\end{array}$ & $\begin{array}{l}\text { Diseño curricular incluyendo los ODS } \\
\text { Capacitación de docentes en Aprendizaje Basado en Problemas (ABP) } \\
\text { Implementación de un modelo educativo basado en ABP } \\
\text { Creación de espacios para discusión del quehacer universitario para lo- } \\
\text { grar los ODS } \\
\text { Contacto permanente con egresados } \\
\text { Evaluación de actitudes de Responsabilidad Social (RS) y aptitudes de } \\
\text { egresados a través de opinión de empleadores } \\
\text { Solicitar la participación de grupos de interés externos en el diseño cu- } \\
\text { rricular }\end{array}$ \\
\hline Investigación & $\begin{array}{l}\text { Impulsar el desarrollo regional, nacio- } \\
\text { nal e internacional a través de la gene- } \\
\text { ración pertinente del conocimiento y } \\
\text { su divulgación }\end{array}$ & $\begin{array}{l}\text { Diseño de Líneas de Generación del conocimiento pertinentes con la pro- } \\
\text { blemática social local y regional, alineadas a los ODS. } \\
\text { Difusión de los resultados de las investigaciones }\end{array}$ \\
\hline Participación Social & $\begin{array}{l}\text { Participar y liderar políticas y progra- } \\
\text { mas de desarrollo socialmente res- } \\
\text { ponsable }\end{array}$ & $\begin{array}{l}\text { Ejecución de diagnósticos integrales y multidisciplinares } \\
\text { Participación en el diseño de agendas de desarrollo local y regional, ali- } \\
\text { neadas a los ODS. } \\
\text { Realización de foros de discusión sobre resultados del desarrollo local y } \\
\text { regional }\end{array}$ \\
\hline $\begin{array}{l}\text { Gestión Organiza- } \\
\text { cional }\end{array}$ & $\begin{array}{l}\text { Crear un ambiente laboral favorable, } \\
\text { ético, justo, con atención al bienestar } \\
\text { de los trabajadores y responsable am- } \\
\text { bientalmente }\end{array}$ & $\begin{array}{l}\text { Sensibilización a los trabajadores para lograr un buen ambiente laboral } \\
\text { Creación de espacios de difusión permanentemente de los ODS en la co- } \\
\text { munidad universitaria } \\
\text { Transparencia en convocatorias y resultados } \\
\text { Diseño de programa de estilos saludables de vida } \\
\text { Evaluación de forma permanente, utilizando métodos cuali-cuantitativos } \\
\text { Evaluaciones externas por grupos de interés }\end{array}$ \\
\hline
\end{tabular}

Nota: Elaboración propia.

\section{Tercer plano}

La evaluación. Estructura, procesos y resultados.

Los resultados obtenidos en el área de la salud abordando los tres enfoques de evaluación de la calidad, la estructura, los procesos y los resultados, han sido muy útiles para que se lleve a cabo de manera integral; por ello, en el modelo de RSU propuesto se introducen en el esquema del modelo para armonizarlo.

Este plano se refiere a la calidad para el logro de los objetivos, aplicación de las estrategiay alcance en las metas en cada una de las funciones sustantivas de la institución. Este planteamiento es consistente con la definición de calidad de la educación planteada por la UNESCO, que la considera "un concepto multidimensional que abarca todas las funciones y actividades institucionales, así como sus recursos, procesos y resultados [...]" (UNESCO \& IESALC, 2019, p. 11).

En la estructura se consideran las características invariantes de cómo se está proporcionando la atención, en el caso que nos ocupa, el proceso de enseñanza aprendizaje. Los procesos se refieren a lo que hacen y cómo lo hacen directivos, personal administrativo y docentes, en su interacción con los estudiantes y grupos de interés externos. Los resultados son los impactos, positivos y negativos, del quehacer de la universidad.

\section{Funciones sustantivas de la Universidad}

Los tres planos del modelo propuesto, que incluyen la filosofía institucional, los objetivos, estrategias, metas y 
la evaluación, adquieren significado cuando convergen en las cuatro funciones sustantivas universitarias y cada herramienta administrativa cumple su función.

Formación. De acuerdo con Vallaeys (2008b), una formación académica socialmente responsable favorece el desarrollo humano y la responsabilidad ambiental, tanto de los estudiantes como de la sociedad en general. En congruencia, el modelo educativo debe fomentar la creatividad, el conocimiento, la solución de problemas y desarrolla habilidades cognitivas, sociales e interpersonales, con lo que los estudiantes, al egresar, estarán en posibilidades de tomar decisiones éticas y generar respuestas a los desafíos que enfrentarán para impulsar un desarrollo social y sustentable en sus diversos planos. Por ello, la recomendación es que se trabajen las diversas asignaturas con un modelo de Educación Basada en Competencias, con preponderancia en Aprendizaje Basado en Problemas (ABP).

Investigación. La investigación socialmente útil y la gestión social del conocimiento conducen el diagnóstico de necesidades sociales del entorno, además de que transmiten los resultados e impulsan el desarrollo humano sostenible.

Participación Social. Una participación social responsable impulsada desde la universidad promueve el desarrollo humano sostenible entre las comunidades.

Gestión organizacional. En alineación con los valores institucionales, debe llevarse a cabo una gestión ética, abierta a la autocrítica, a la democracia, que aprende diariamente de todos sus miembros, donde existan canales de comunicación efectivos, con procesos de contratación transparentes. También incluye la atención a los trabajadores, ya sean docentes o trabajadores administrativos, a quienes la universidad facilita su desarrollo profesional, está pendiente de que se realicen acciones que mejoren el ambiente laboral y que alcancen a las familias de sus trabajadores; además, la gestión atiende la salud y promueve estilos de vida saludables en la comunidad y sobre todo, sensibiliza de manera permanente a todos sus miembros para que sean ejemplo de cómo vivir en valores.

Congruente con sus valores, la universidad responde por el impacto que produce en el medio ambiente y de manera permanente introduce mejoras para minimizarlo. Se ocupa de mejorar la disposición de su agua residual y la basura que se genera y continuamente busca incrementar el uso de energías renovables, además de reciclar todos los elementos que pueda.

En este rubro se considera también la selección y evaluación de los proveedores, a quienes les pide evidencias de su Responsabilidad Empresarial, con lo que se genera una dinámica positiva que dará resultados en el tema ambiental para toda la sociedad. El marketing de la universidad hacia el exterior se basa en resultados reales, sin afán de maquillar cifras para lograr mayores ingresos o presupuestos (Vallaeys, 2016).

Interrelación de los elementos incluidos en el modelo integrador ODS-RSU-Planeación Estratégica

Una gestión socialmente responsable de la universidad se debe reflejar en la organización misma y en las otras tres funciones sustantivas, como son: la formación de los estudiantes, la investigación atendiendo al modelo epistemológico que subyace y en la participación social para el desarrollo sostenible. De esta manera el impacto se evidencia en todas las funciones, las que se enriquecen, creándose una sinergia que facilita las actividades y la vida cotidiana, con lo que se educa a todos los miembros (Vallaeys, 2016).

Así también, las posibles relaciones entre los elementos 
de la triada que establece la evaluación de la calidad deben esclarecerse, esto es, entre los procesos y los resultados, así como entre la estructura y los procesos, para lo cual se utiliza un abordaje socio-organizativo, que abarca muchas facetas del fenómeno en el tránsito gradual de la estructura al proceso y de éste al resultado (Donabedian, 1986/2001b).

Tomando en cuenta lo anterior, el esquema del modelo propuesto en la figura 4, muestra la interrelación dinámica de todos los elementos con el propósito de que las actividades de la universidad apoyen para el logro de los ODS, de ahí que estos deben inspirar la misión, la visión y los valores institucionales donde se expresa lo que la institución es y lo que quiere ser en un marco de RSU. Hacia dónde se dirige se plantea en los objetivos y cuantifica en las metas, mientras que el cómo lo va a hacer se expresa en las estrategias, todo ello diseñado para la formación, investigación, participación social y gestión organizacional.

Para efectos de evaluación y control, se diseñan o eligen los indicadores de estructura, procesos y resultados en cada de las funciones mencionadas. Así, los momentos de la planeación y evaluación quedan unidos por las dimensiones y si se modifica algo, su propia dependencia hace que se dinamice el proceso para realinearse (figura 4).

\section{Figura 4}

Interrelación de los elementos del modelo de RSU propuesto

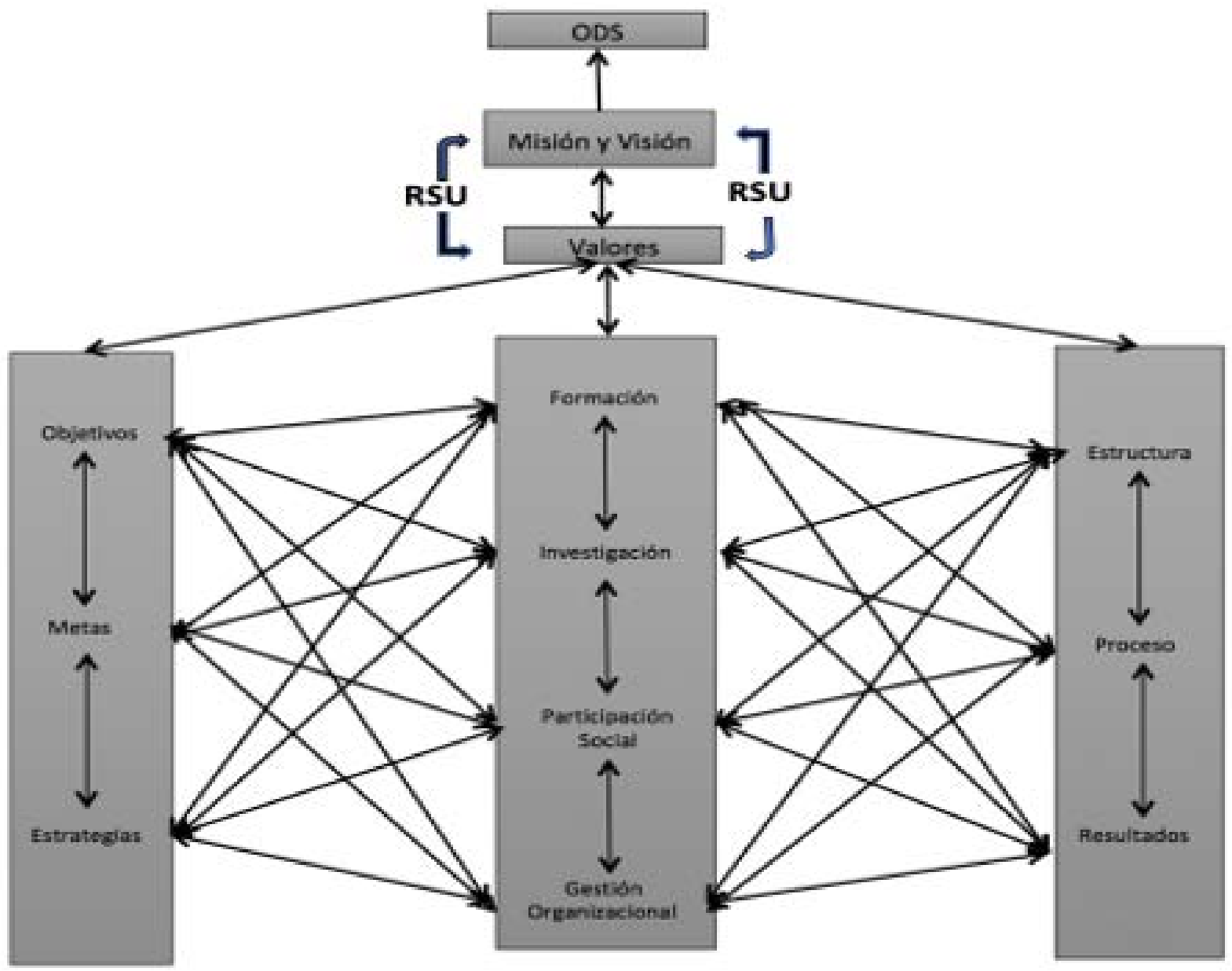




\section{| Conclusiones}

A partir de 2016, la ONU puso en marcha una transformadora agenda 2030 para el Desarrollo Sostenible que fue adoptada por los países miembros para lograr 17 objetivos establecidos como resultado del análisis de las profundas desigualdades económicas y sociales entre las regiones el mundo.

Para avanzar en el cumplimiento de los ODS se requiere del esfuerzo conjunto de todos los sectores productivos, donde el Sector educativo y, especialmente las IES, tienen un compromiso ineludible con su logro, ya que está visto que el paso de los estudiantes por la universidad es una magnífica oportunidad para sensibilizarlos y educarlos mediante programas y proyectos académicos consistentes, que además de formarlos, estén orientados al bienestar colectivo, el cuidado del ambiente y los recursos naturales en un marco de valores éticos.

El reto es inmenso, pues al modelo tradicional arraigado en la comunidad universitaria se suman las nuevas formas de producción del conocimiento, las presiones de actores externos derivadas de las agendas de desarrollo, los recursos siempre insuficientes y la poca claridad del qué y cómo aportar de manera efectiva a partir de la academia.

Como respuesta a lo anterior, en la última década, desde las IES y organizaciones latinoaméricas, han surgido modelos de RSU que se han aplicado y, derivado de la evaluación de sus resultados, han evolucionado en el tiempo proporcionando elementos teóricos y filosóficos cada vez más consistentes. Sin embargo, el tema de los ODS no se declara abiertamente en los modelos, aunado a que, con frecuencia, su esquematización adolece de no presentar todos los elementos involucrados, es incompleta o definitivamente no se realiza. Por ello, se hace necesario proponer un modelo de RSU cuyo objetivo sea abonar al logro de los ODS, que sea claro, pertinente, cuya representación gráfica proporcione coherencia y muestre las relaciones de los elementos que lo integran.

El modelo de integración ODS-RSU-Planeación estratégica propuesto hace referencia explícita a los ODS en dos figuras; una con sus elementos y otra con las relaciones que se establecen entre ellos. La primera considera tres planos que interactúan entre sí y son dinámicos. En el primero, para destacar cuál es el rumbo a seguir, centra a los ODS, rodeados por la misión, visión y valores institucionales, en cuya redacción debe quedar plenamente expresada la RSU para que sea inobjetable que forma parte de la filosofía institucional. En un segundo plano del modelo se presentan tres elementos clave de la planeación estratégica institucional, los objetivos, estrategias y metas. El tercero considera la triada de la evaluación de la calidad dirigida a la estructura, los procesos y los resultados. Los tres planos son dinámicos y están referidos a las cuatro funciones sustantivas de la universidad.

Una segunda figura da cuenta de las relaciones que se establecen, las cuales son multiples, simultáneas y dinámicas. En el esquema se aprecia cómo todos los elementos apuntan hacia los ODS. Las múltiples flechas indican la interrelación de sus elementos, de tal manera que el cambio en uno afecta varios puntos a la vez.

El modelo necesita del diseño de indicadores que sean claros, precisos y aplicables a cada una de las funciones sustantivas para poder monitorear y evaluar las prácticas y las metas que hubiere establecido la institución en diferentes periodos de tiempo, como pueden ser, la inclusión de la RSU en su marco filosófico, la normatividad, la planeación y, además, cómo se operacionaliza en los planes y programas de estudio, en los programas de trabajo de todas las áreas administrativas, por citar algunas dimensiones. 
Este trabajo tiene tres limitaciones. La primera es que no se ha probado el modelo, por lo que se desconoce hasta qué punto apoya en clarificar los elementos y planos que intervienen en él y las relaciones entre los ODS, la RSU y la planeación estratégica institucional. La segunda es que debe aplicarse en el momento que se realice un nuevo Plan de Desarrollo Institucional (PDI), lo que ocurre cada cambio de administración, y la tercera es que, para poder aplicarlo, las nuevas autoridades deberían aprobarlo, de ahí que tendrían que estar previamente sensibilizados al cambio hacia un nuevo paradigma y convencidos de que las IES necesitan asumir un papel protagónico en la contribución a la agenda mundial 2030.

De no haber un cambio en el paradigma actual de las IES, que las comprometa a contribuir al logro de los ODS, continuarán sus operaciones centradas en sus propios intereses sin obtener resultados de gran calado para la sociedad. Por ello, es importante tender puentes de comunicación efectiva con todos los actores para identificar los retos, las oportunidades y desplegar las estrategias que sean necesaria para dar luz a una hoja de ruta comprometida responsablemente con la sostenibilidad.

La discusión, adaptación y aplicación del modelo propuesto, cuya principal característica es armonizar esquemáticamente los elementos que participan en la RSU, colocando en el centro a los ODS, además de integrar y relacionar a la RSU desde la declaración de principios con la planeación estratégica, las funciones sustantivas universitarias y la evaluación estratégia desde tres enfoques, puede ser una buena opción para sumar a las universidades al llamado realizado por la ONU a poner fin a la pobreza, proteger el planeta y garantizar que todas las personas gocen de paz y prosperidad para el año 2030.

\section{| Referencias}

Asociación Nacional de Facultades y Escuelas de Contaduría y Administración [ANFECA]. (2017). Modelo de Responsabilidad Social de ANFECA. Universidad Veracruzana. https://bit.ly/RS ANFECA

Asociación de Universidades Confiadas a la Compañía de Jesús en América Latina [AUSJAL]. (2009). ¿Cómo concebir la RSU en las Universidad de AUSJAL? En Gargantini y Zaffaroni (Eds.), Políticas y sistema de autoevaluación y gestión de la responsabilidad social universitaria en AUSJAL. AUSJAL. https://bit.ly/ ausjal2009

Calle-Ramírez, D. C. \& Santacruz-Moncayo, T. C. (2011). Modelo de responsabilidad social universitaria aplicado en la Universidad Politécnica Salesiana Sede Cuenca [Tesis de Maestría, Universidad Politécnica Salesiana]. https://bit.ly/RSU Salesiana

Castilla, J. C. (2015). Tragedia de los recursos de uso común y ética ambiental individual responsable frente al calentamiento global. Acta bioethica, 21(1), 65-71. https://bit.ly/CASTILLA2015

Comisión Económica para América Latina y el Caribe [CEPAL]. (2009). Guía metodológica: Diseño de indicadores compuestos de desarrollo sostenible. CEPAL. https://cutt. ly/5CzmABK

Cruz Álvarez, J. G., Rositas Martínez, J. \& García Mendoza, J. (2013, 2-4 de Octubre). Responsabilidad Social Empresarial: Investigación empírica - exploratoria sobre los conceptos de RSE y RSU [Ponencia]. XVIII Congreso Internacional de Contaduría, Administración e Informática, México, D.F. https://bit.ly/CRUZ2013

De Dios-Pérez, J. \& Vallaeys, F. (2016). Universidad Autónoma de Chihuahua [UACH]. Modelo de Responsabilidad Social Universitaria. En J. De Dios-Pérez \& F. Vallaeys (Ed.), Prácticas y modelos de responsabilidad social universitaria en México: proceso de transformación de la universidad. ANUIES. https://bit. ly/DEDIOS2016 
Donabedian, A. (2001a). La calidad de la atención sanitaria: ¿De quién es la responsabilidad? Revista Calidad Asistencial, 16(1), S29-S38. https://bit.ly/DONAB2001

Donabedian, A. (2001b). Gestión del conocimiento y calidad asistencial. La investigacion sobre la calidad de la atención médica. Revista de calidad asistencial, 16(1), S63-S66. https://bit.ly/DONAB2001

Deutsche Gesellschaft für International Zusammenarbeit [GIZ] GmbH. (2015). Cooperation Management for Practitioners: Managing Social Change with Capacity WORKS. Gabler Verlag. https://doi.org/g9v7

Forero-Jiménez, M. Y. (2019). Modelo de responsabilidad social universitaria: Una propuesta para las instituciones colombianas. Revista de Investigación, Desarrollo e Innovación, 9(2), 249-260. https://doi.org/ g9v8

Gaete, R. (2015). La responsabilidad social universitaria desde la perspectiva de las partes interesadas: Un estudio de caso. Actualidades Investigativas en Educación, 15(1). https://bit.ly/GAETE2015

Gaete, R. \& Álvarez-Rodríguez, J. (2019). Responsabilidad social universitaria en Latinoamérica. Los casos de URSULA y AUSJAL. Revista Actualidades Investigativas en Educación, 19(3), 1-27. https://bit.ly/GAETE2019

Hax, A.C. \& Majluf, N.S. (1984). The Corporate Strategic Planning Process. Informs Journal on applied analytics, 14(1), 47-60. https://doi.org/cmdbn2

Katayama-Omura, R. (2014). Modelos de Responsabilidad Social Universitaria. Gestión en el Tercer Milenio, 17(33), 39-44. https://doi.org/g9v9

Navarro, I. (2011). La Planeación y Gestión Estratégica de la Responsabilidad y el Compromiso Social: Un Reto para las Universidades Latinoamericanas. Boletín IESALC Informa. https://bit.ly/Navarro2011

Kouatli, I. (2019). The contemporary definition of university social responsibility with quantifiable sustainability. Social Responsibility Journal, 15(7), 888-909. https://bit. ly/KOUATLI2019

Organización de las Naciones Unidas [ONU]. (2016a).
Objetivos de Desarrollo Sostenible. ONU. https://bit.ly/ ONU2016a

Organización de las Naciones Unidas [ONU]. (2016b). La agenda para el Desarrollo Sostenible. ONU. https://bit. ly/ONU2016B

Organización de las Naciones Unidas para la Educación, la Ciencia y la Cultura [UNESCO]. (2009). Conferencia mundial sobre la educación superior - 2009: La nueva dinámica de la educación superior y la investigación para el cambio social y el desarrollo. Revista da Avaliação da Educação Superior (Campinas), 14(3), 755766. https://bit.ly/ONU2009

Organización de las Naciones Unidas para la Educación, la Ciencia y la Cultura [UNESCO] \& Instituto Internacional para la Educación Superior en América Latina y el Caribe [IESALC]. (2019). Plan de Acción CRES 2018-2028. IESALC. https://bit.ly/IESALC2019

Ramírez, R. (2017). Hacia un cambio en la matriz cognitiva en el sistema de ciencia, tecnología e innovación de América Latina y el Caribe. Revista Educación Superior y Sociedad (ESS), 27(27), 17-50.. https://bit.ly/ IESALC2017

Schantz, N. M., Charles, A. \& Copestake, J. (2021). The Sustainable Development Goals and the University of Bath: An Opportunity. University of Bath. https://doi. org/g9wb

Unión de Responsabilidad Social Universitaria Latinoamericana [URSULA]. (2019). Investigación Continental URSULA: estado del arte de la Responsabilidad Social Universitaria (RSU) en América Latina - 2018. URSULA. https://bit.ly/URSULA2018

Universidad Autónoma de Yucatán [UADY]. (2014). Plan de Desarrollo Institucional. 1a Agenda Estratégica. Acción 10: Modelo de Responsabilidad Social Universitaria. UADY. https://bit.ly/UADY2014

Universidad Autónoma Metropolitana [UAM]. (2013). Modelo de Responsabilidad Social Universitaria. UAM. https://bit.ly/UAM2013

Universidad Construye País. (2002). Observando la 
Responsabilidad Social Universitaria. Documento de trabajo preparado por equipo coordinador del proyecto Universidad. Construye País. https://bit.ly/UCP2004

Universidad de San Ignacio de Loyola [USIL]. (2018). Modelo de Responsabilidad Social Universitaria. USIL. https:// bit.ly/USIL2018

Uribe-Macías, M. E. (2016). Propuesta del modelo de gestión de la RSU para la Universidad del Tolima. En S. Fajardo y L. Michel (Eds.), Responsabilidad social de las organizaciones (RSO): aportes teóricoprácticos para lograr los Objetivos de Desarrollo sostenible en América Latina (pp. 209-226). Ediciones USTA. https://bit.ly/ RSO SIRSO

Valarezo-González, K. \& Túñez-López, J. M. (2014). Responsabilidad Social Universitaria. Apuntes para un modelo de RSU. Revista de comunicación, 13, 84117. https://bit.ly/VALARE2014

Vallaeys, F. (2008a). El movimiento de responsabilidad social de la universidad: Una comprensión novedosa de la misión universitaria. Educación Superior y Sociedad, 13(2). https://bit.ly/IESALC08A

Vallaeys, F. (2008b). Responsabilidad Social Universitaria: Una nueva filosofía de gestión ética e inteligente para las universidades. Revista Educación Superior y Sociedad (ESS), 13(2),19-220. https://bit.ly/VALLAEYS2008B

Vallaeys, F., De la Cruz, C., \& Sasia, P. M. (2009). Responsabilidad social universitaria: Manual de primeros pasos. McGraw-Hill. https://bit.ly/VALLAE2009

Vallaeys, F. (2014). La Responsabilidad Social Universitaria: Un nuevo modelo universitario contra la mercantilización. Revista Iberoamericana de Educación Superior, 5(2), 105-117. https://bit.ly/VALLAEYS2014

Vallaeys, F. (2016). Introducción a la Responsabilidad Social Universitaria (RSU). Ediciones Universidad Simón Bolivar. https://cutt.ly/GCzQ5ag

Vallaeys, F. (2021). Manual de Responsabilidad Social Universitaria. El modelo URSULA: estrategias, herramientas, indicadores. URSULA. https://bit.ly/ VALLEYS2021
Winkler, M. I., Alvear, K., Olivares, B. \& Pasmanik, D. (2012). Lo ético es transversal y cotidiano: Dimensiones éticas en la formación y práctica en psicología comunitaria. Acta bioethica, 18(2), 237-245. https:// doi.org/g9wc 